\title{
Modelación y Tecnología en el Estudio de la Tasa de Variación Instantánea en Matemáticas
}

\author{
Jhony A. Villa-Ochoa ${ }^{(1)}$, Difariney González-Gómez ${ }^{(2)}$ y Jaime A. Carmona-Mesa(1) \\ (1) Universidad de Antioquia, Facultad de Educación, Grupo MATHEMA-FIEM, Calle 67 No. 53-108, \\ Oficina 9-415, Medellín, Colombia (e-mail: jhony.villa@udea.edu.co; jandres.carmona@udea.edu.co) \\ (2) Universidad de Antioquia, Facultad Nacional de Salud Pública, Grupo Demografía y Salud, \\ Calle 67 No.53-108, Medellín, Colombia (e-mail: difariney.gonzalez@udea.edu.co)
}

Recibido May. 24, 2017; Aceptado Jul. 21, 2017; Versión final Sep. 6, 2017, Publicado Abr. 2018

\begin{abstract}
Resumen
Se presentan los resultados de un estudio destinado a reconocer las contribuciones que los contextos y las tecnologías digitales ofrecen a la comprensión de la tasa de variación instantánea como una manera de aproximarse a la derivada en un punto en el estudio de las matemáticas. A través de documentos, entrevistas y videograbaciones pudo observarse que los fenómenos modelados y las tecnologías digitales no son neutros en la comprensión de este objeto matemático. Los resultados muestran que las situaciones en las cuales la tasa de variación puede asociarse a magnitudes, en contextos y experiencias propias de los sujetos, contribuye a pasar de la comprensión de la tasa de variación media a la comprensión de la tasa de variación instantánea. El estudio sugiere la necesidad de diseñar ambientes que promuevan que los estudiantes se enfrenten a experiencias de modelación con tecnología en la que los objetos matemáticos puedan tener diversidad de significados e interpretaciones.
\end{abstract}

Palabras clave: modelación matemática; tecnologías digitales; tasa de variación; derivada

\section{Modelling and Technology in the Study of the Instantaneous Rate of Change in Mathematics}

\begin{abstract}
This paper presents the results of a study aimed to recognize the contributions that contexts and digital technologies offer to the understanding of the instantaneous rate of change as a means for approximating to the concept of derivative in the study of mathematics. It was possible to observe through documents, interviews and video recordings, that phenomena modelled and digital technologies are not neutral in the understanding of this mathematical object. The results show that situations in which the rate of change may be associated with magnitudes, in contexts and experiences of the subjects, contribute to move from the understanding of the average rate of change to the understanding of the instantaneous rate of change. This study suggests that it is necessary to design environments that encourage students to face modelling experiences with technology in which mathematical objects could have a diversity of meanings and interpretations.
\end{abstract}

Keywords: mathematical modelling; digital technologies; rate of change; derivative 
Modelación y Tecnología en el Estudio de la Tasa de Variación Instantánea en Matemáticas Villa-Ochoa

\section{INTRODUCCIÓN}

Existe un creciente interés por el desarrollo de investigaciones que incluyan la modelación matemática y las tecnologías digitales en los procesos formativos en matemáticas (Borba y Villarreal, 2005). La conjunción de la modelación con las tecnologías digitales está presente en diversos estudios internacionales. Por ejemplo, Suárez (2014) mostró cómo los estudiantes pueden construir relaciones funcionales de fenómenos de cambio a través de sensores y calculadoras, Soares y Borba (2014) argumentaron que un software como el Modellus permite llevar a cabo un proceso de análisis de modelos matemáticos que intervienen en la biología, Daher y Shahbari (2015) y Rodríguez y Quiroz (2016) mostraron que los procesos de modelación se reorganizan cuando se desarrollan tareas y experimentos con tecnología.

Un aspecto común en los anteriores trabajos es que las tecnologías digitales cumplen un rol fundamental para la obtención y análisis de datos, producción de modelos o la validación y análisis de los mismos. Otro aspecto recurrente en la mayoría de estos estudios es la posibilidad de modelar fenómenos de variación a través de conceptos del análisis matemático (funciones, derivadas, ecuaciones diferenciales, entre otros). Este interés converge con los planteamientos de Tall (2009), quien resalta la importancia de los aspectos dinámicos de la matemática y el papel del software en la reproducción de efectos visuales del cálculo. Este autor también señala que el cálculo está compuesto por conceptos dinámicos, por ejemplo: el deseo de cuantificar las cosas que cambian (el concepto de función), la razón en la cual cambian (derivada), la manera en la que se acumulan (la integral) y las relaciones entre ellas (teorema fundamental del cálculo y la solución de ecuaciones diferenciales).

La variación ha sido un aspecto central en el surgimiento histórico de conceptos del cálculo (Gordillo y PinoFan, 2016). También ha sido de interés diversos estudios que se preocupan por su presencia y comprensión en la Educación matemática (Vrancken y Engler, 2014; Ärlebäck et al., 2013, Herbert y Pierce, 2011, 2012). Ärlebäck et al. (2013) analizaron la interpretación que hacen los estudiantes de los modelos y su relación con la interpretación de la tasa de variación media. En relación con este concepto, Herbert y Pierce (2011) informaron que las habilidades de los estudiantes para trabajar productivamente con representaciones en un contexto no necesariamente se trasladan a otras representaciones y contextos. En un estudio con estudiantes de primer año de ingeniería, Vrancken y Engler (2014) informaron que a través de un conjunto de situaciones de variación la derivada emerge a partir de la necesidad de cuantificar los cambios en un instante. También con estudiantes de ingeniería, Báez et al., (2017) mostraron evidencias de que una propuesta centrada en tareas de variación y cambio apoya el desarrollo del pensamiento variacional en contextos propios de estos profesionales. En un contexto diferente, Villa-Ochoa (2012) ofreció evidencia de que las representaciones que los estudiantes usan influyen en la manera en que razonan con la covariación; también mostró que la transición de la comprensión de la tasa de variación media a la tasa de variación instantánea no es inmediata. Los contextos y las representaciones están presentes en la actividad matemática; sin embargo, no siempre tienen los mismos usos ni cumplen los mismos roles en el razonamiento de los estudiantes. En ese sentido, este artículo reporta parte de los resultados de un estudio más amplio que se preocupó por la comprensión de la tasa de variación. En particular, este artículo se centra en reconocer las contribuciones que los contextos y las tecnologías digitales ofrecen a la comprensión de la tasa de variación instantánea.

\section{MODELACIÓN MATEMÁTICA EN EDUCACIÓN MATEMÁTICA ¿QUÉ SIGNIFICÓ EN ESTE ESTUDIO?}

De manera general, la modelación puede comprenderse como una práctica de articulación entre dos entes, uno llamado modelo que actúa en otro llamado lo modelado. La intervención en lo modelado es diversa, por ejemplo, para la predicción, el diagnóstico o la evaluación de aspectos del ente modelado (Arrieta y Díaz 2015). En la literatura referida a la Educación Matemática no existe una comprensión homogénea acerca de la modelación matemática ni de las implicaciones en el aula (Kaiser, Sriraman, 2006); la mayoría de los estudios coinciden en denominarla como una interacción entre el "mundo real" (ente modelado) y las matemáticas (ente modelador) (Blum, 2011). Sin embargo, más allá de reportar experiencias realísticas y auténticas, algunos autores señalan que conviene centrar también la atención al interior de las matemáticas escolares para enriquecer la práctica actual de la modelación matemática con el fin de aprender matemáticas y apropiarse de herramientas útiles de una manera significativa, incluso cuando las prácticas profesionales "reales" aún no están incorporadas de forma plena (Arcaví, 2008).

Uno de los aspectos diferenciadores entre las tareas y procesos de modelación es la noción de "realidad" (Villa-Ochoa, 2015), incluso, en el sentido de Arcavi (2008) los entes modelados no tienen que articularse de forma necesaria a fenómenos extramatemáticos, por lo tanto, situaciones y fenómenos de la misma matemática pueden ser susceptibles de ser modelados, de esa manera, toda práctica matemática es una práctica de modelación (Bosch et al., 2006). 


\section{EL ESTUDIO}

Ya que los significados y comprensiones de los objetos matemáticos guardan estrecha relación con las acciones, prácticas, razonamientos y demás actividades que se involucran en la resolución de problemas, este estudio se desarrolló a través de una metodología cualitativa, en particular, se utilizó el método de estudio de casos. Para Yin (2009), un estudio de casos posibilita una inmersión profunda y exhaustiva en un objeto delimitado, en este caso, en un fenómeno educativo.

En el estudio participaron cuatro estudiantes de un curso de pre-cálculo de un programa de ingeniería de una universidad pública en Medellín, Colombia. Las estudiantes se nombran bajo los seudónimos Cristina, Marcela, Estefanía y Alexandra, quienes decidieron participar de forma voluntaria y se comprometieron a participar de ocho sesiones con una intensidad de dos horas cada una. Las sesiones fueron orientadas por uno de los investigadores autores de este artículo. En los diálogos que se presentarán más adelante, se le nombrará como "investigador". En las sesiones las estudiantes estaban dedicadas al estudio de tareas referidas a la modelación de fenómenos de covariación. Las tareas tenían como propósito analizar el comportamiento de variables, reconocer patrones y establecer mecanismos para construir modelos que representaran la covariación.

Las tareas se desarrollaron con el uso del software GeoGebra y Modellus. La primera tarea se denominó "rectángulo inscrito" y se retomó del trabajo de Villa-Ochoa (2012). Ésta se diseñó en el software GeoGebra y se construyó una herramienta que permitiera visualizar la tasa de variación a través de un conjunto de segmentos que representaban el cociente incremental (tasa de variación) en diferentes puntos de una función. En la figura 1 se presentan las principales características de la tarea.

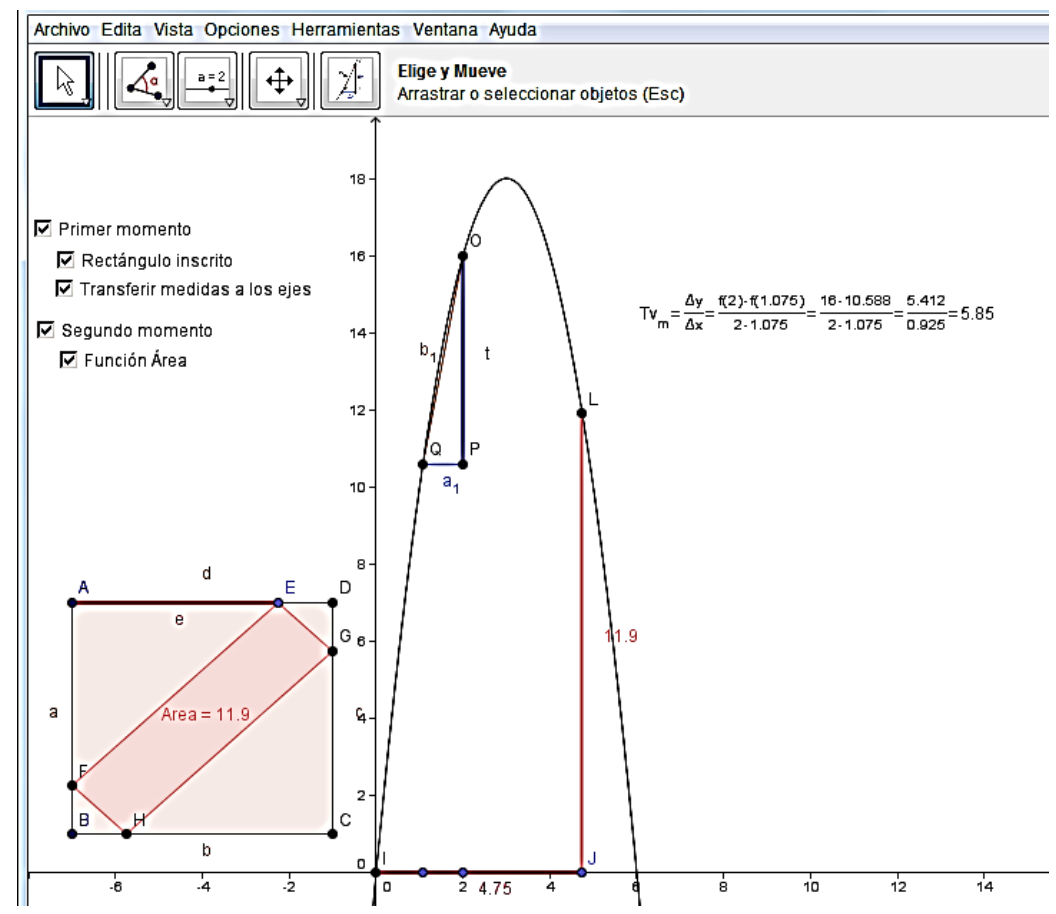

Fig. 1: Ambiente de la tarea 1

La segunda tarea se denominó "velocidad y aceleración". En ella se utilizó el software Modellus para analizar las relaciones entre recorrido, velocidad y aceleración de un móvil de acuerdo con la misma función cuadrática descrita en la tarea 1. Esta segunda tarea se desarrolló a través de la vista dinámica, tabular y gráfica del software. La ventana que contenía los modelos matemáticos permaneció oculta. Para la tercera tarea se propuso nuevamente el uso del GeoGebra; esta tarea consistió en estudiar la función Tasa de variación en cualquier función, a través de una herramienta que generalizaba los triángulos construidos en la tarea 1 a múltiples triángulos en un intervalo. La cuarta tarea consistió en analizar la variación en la descarga de un archivo de internet. Para ello se presentó a las estudiantes un video en el que se registraba el proceso de descarga. La datos de este estudio fueron los documentos elaborados por las estudiantes en la solución de las tareas propuestas, entrevistas, grabaciones de audio y registro en video de las acciones que realizaron en los computadores, este último registro se realizó con un demo del software Camtasia. El análisis se enfocó en todos aquellos datos que dieran cuenta de qué y cómo las estudiantes comprendían la tasa de variación; a partir de ello, la atención se orientó al rol que tuvo la modelación y la tecnología en dichas comprensiones. 


\section{LA TASA DE VARIACIÓN INSTANTÁNEA EN UN AMBIENTE DE MODELACIÓN CON TECNOLOGÍA}

El presente artículo se fundamenta en la participación de las estudiantes durante las dos primeras tareas. En su primer acercamiento, las estudiantes se comprometieron con el reconocimiento de las cantidades variables y constantes, luego determinaron y describieron relaciones de covariación entre ellas. Al igual que en los trabajos de Carlson et al. (2003), Cabezas y Mendoza (2016) y Villa-Ochoa (2012), las participantes reconocieron características de la dirección de la covariación (v.g. el área aumenta y luego disminuye). Seguidamente, el trabajo se centró en cuantificar el cambio y en construir un modelo matemático para representarlo; para ello, las estudiantes construyeron un punto móvil cuya abscisa fue la distancia AE y la ordenada el área del rectángulo EFHG (Ver Fig.1). Al recrear el movimiento del segmento AE, el punto "dejó el rastro" y con base en él, usaron la herramienta "lugar geométrico". Como una manera alternativa de construir el modelo, el investigador sugirió representar con la variable $x$ el segmento $A E$, las estudiantes lograron determinar la función área $A(x)=2 x(6-x)$ con base en las relaciones geométricas entre los diferentes segmentos. En la expresión algebraica 6 corresponde a la longitud del lado del cuadrado.

Dado que el objeto central fue el estudio de la tasa de variación, el investigador cuestionó a las participantes acerca de cómo y cuánto cambia la función en determinados "momentos" del recorrido. Ellas centraron la atención en las diferencias en ciertos intervalos y mencionaron aspectos cualitativos como "crece cada vez más lento pero hasta la mitad'. Después, el investigador les sugirió explorar la herramienta Tasa de variación que se había diseñado en el software. El investigador pidió centrar la atención en $x=2$. Las estudiantes utilizaron la herramienta para calcular y representar la tasa de variación en diferentes intervalos, por ejemplo: [1.5, 2], [1.7, 2], [1.9, 2], [1.99, 2]. En la Fig. 2 se muestra la construcción hecha por Marcela y Cristina.

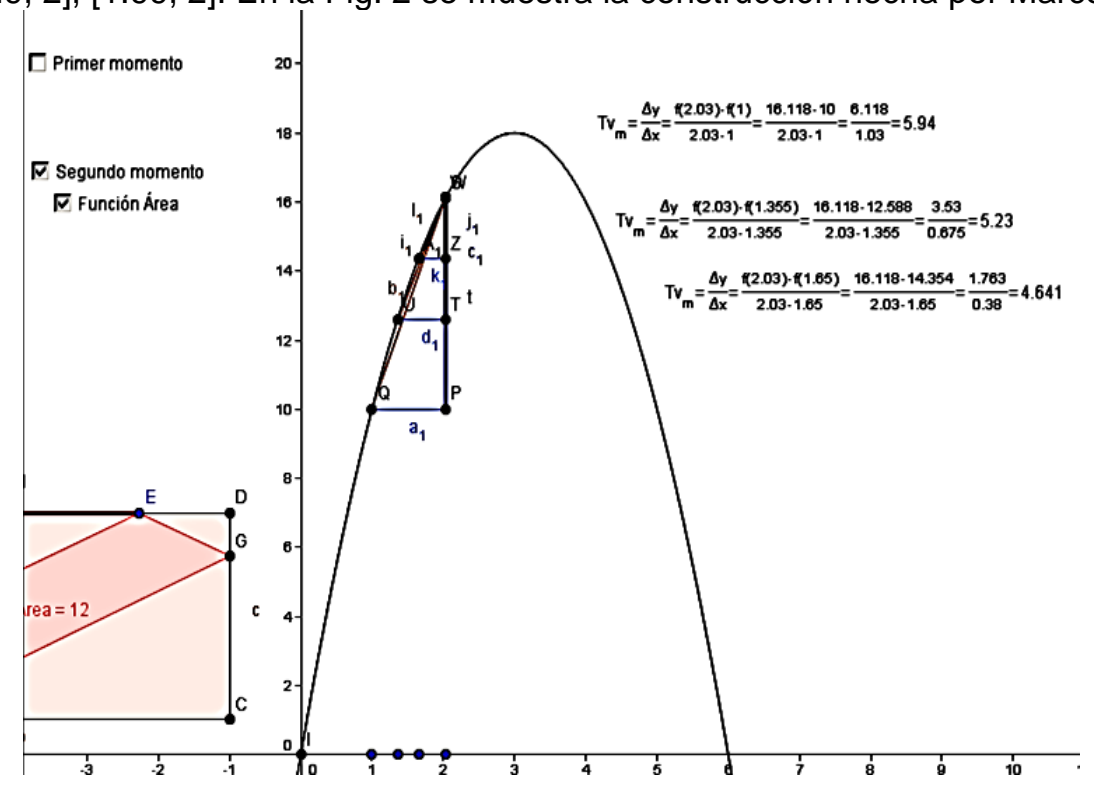

Fig. 2: Imagen de la aplicación de la herramienta Tasa de variación, por Marcela y Cristina.

Para responder la pregunta acerca de cómo cambia la tasa de variación en $x=2$, las estudiantes se apoyaron en cálculos aritméticos proporcionados por la herramienta en cada intervalo. Al ver la secuencia de resultados, expresaron que la tasa de variación decrece. El investigador interpeló de nuevo preguntando ¿Hasta dónde decrece?, a lo que Estefanía respondió hasta uno; el investigador les pidió argumentos para validar o refutar esa respuesta. Las estudiantes continuaron usando la herramienta Tasa de variación para calcular los valores de la tasa en intervalos cada vez más pequeños (con 2 en el extremo derecho del intervalo). El investigador preguntó de nuevo: ¿Seguras que decrece hasta uno? A lo que Cristina y Estefanía respondieron con gestos que indicaban su respuesta negativa. Enseguida, el investigador preguntó: ¿Cuál es el valor al que nos acercamos cuando el punto se aproxima a 2? Las cuatro estudiantes respondieron cuatro, de forma simultánea. Este hecho se convierte en evidencia de la presencia de una imagen del concepto de límite, el cual fue observado como "tendencia". En este episodio, estas imágenes del límite se dieron a partir de la regularidad encontrada en la secuencia aritmética de puntos que proporcionaba el software a través de la herramienta Tasa de variación.

Las estudiantes continuaron moviendo los puntos para construir intervalos cada vez más pequeños y, en una de esas oportunidades, ocurrió que dos extremos del intervalo se superpusieron lo que generó que la expresión en el texto dinámico mostrara $\Delta y / \Delta x=0 / 0=$ ? Este hecho propició que Marcela creara una idea de límite como una "tendencia", que en el caso de la estudiante fue supuesto pero no de existencia. El siguiente diálogo es evidencia de esta interpretación. 
Investigador: Entonces, ¿Cuál es la tasa de variación en 2?

Marcela: ¡No existe!

Investigador: ¿Por qué no existe?

Marcela: $\quad$ ¿Porque cero sobre cero no existe!

Estefanía: ¡Es indeterminado!

Investigador: Y entonces, ¿Qué significa ese cuatro que encontraron? [Refiriéndose al valor del límite inferido por medio de la aproximación].

Marcela: $\quad$ Pero dijimos que daba cuatro porque nos aproximábamos, pero no es cuatro, porque en dos se anula.

Las demás compañeras se limitaron a escuchar y al observar la pantalla del computador concordaron con Marcela y Estefanía. Frente a este hecho es posible generar al menos dos interpretaciones: por un lado, las estudiantes estaban centrando su razonamiento en identificar "el patrón" que ofrecía los cálculos que proporcionaba el software y, por otro, la noción de límite se vinculó con el cálculo aritmético de los valores en un intervalo y no con la "tendencia" que resulta a medida que se generan aproximaciones. Centrar la atención en el cálculo ofrecido por el software pudo conllevar a que las estudiantes no diferenciaran el cálculo de la tasa de variación en un intervalo y el cálculo de ésta en un punto.

En la entrevista individual que se realizó posterior a la experiencia, el investigador preguntó de nuevo por el valor que habían encontrado de la tasa de variación en x=2. Marcela expresó: de 2 a... [Pretendía que el profesor completara su oración]. Esta respuesta muestra que la tasa de variación [instantánea] seguía asociada solo a su comprensión en un intervalo [tasa de variación media]. El profesor interpeló a Marcela y le aclaró que requería la tasa de variación en dos, a lo que ella respondió: $A h$, en 2 daba infinito, daba indeterminado. Al pedirle argumentos al respecto, ella manifestó: Porque... Porque por estas restas daba 0 sobre 0. Porque cuando el área está,..., el área es,..., porque cuando el segmento está en 2, el área es 16 y si lo vamos a comparar, pues, sí... ¡No me acuerdo! [Silencio] Daba indeterminada, porque este segmento... [Señaló el segmento variable del cuadrado]. Marcela, al igual que sus compañeras, a pesar de haber usado la noción de límite como "tendencia", evocó de nuevo la idea de la tasa de variación en un intervalo tal y como se usó en el software.

Las respuestas de Marcela dejaron en evidencia que existen estudiantes para quienes la tasa de variación se asocia a la idea de límite como "tendencia", "valor supuesto" o "tendencia sin llegar". Por lo tanto, requieren de experiencias diferentes que promuevan otras ideas acerca de la aceptación de la existencia de la tasa de variación en un punto. Para atender a este tipo de necesidades, en el software Modellus se recreó un contexto del movimiento de un vehículo (tarea 2). Al igual que en la tarea 1, la modelación inició con el reconocimiento de las cantidades que intervienen en el movimiento, la abstracción de relaciones y la construcción de un modelo que representara la posición del móvil en función del tiempo. El modelo que las estudiantes lograron construir fue $x(t)=2 t(6-t)$.

A partir de este modelo, el investigador sugirió a las participantes comparar el movimiento y los modelos de las tareas 1 y 2 . Los hallazgos en este aspecto muestran que, a pesar de que en ambos casos se estudió la misma función matemática y se hizo un análisis de la tasa de variación de forma semejante, las estudiantes no establecieron una correspondencia entre las características de ambas tareas. El investigador escribió en la pizarra las expresiones $A=2 x(6-x)$ y $x=2 t(6-t)$, y generó un diálogo para promover en las estudiantes una comparación entre ambos fenómenos y expresiones, sobre sus diferencias y semejanzas. A excepción de Cristina, las demás estudiantes lograron concluir que: El incremento de $A$ sobre incremento de $x$, [en la situación "rectángulo inscrito"] es análoga a "triangulito [incremento] de $x$ sobre incremento de t". Simultáneamente el investigador escribió en el tablero la ecuación $\Delta x / \Delta t$. Ante esta respuesta, el investigador preguntó mientras señalaba el cociente incremental escrito en el tablero: ¿Qué sería el cambio de la distancia con respecto al cambio del tiempo? A lo que Alexandra y Marcela respondieron al unísono: La velocidad. El investigador replicó de nuevo: ¿Qué es la velocidad? Y Marcela responde: La relación entre la distancia y el tiempo, Alexandra complementó diciendo: la variación de la distancia con respecto a la variación del tiempo. Las evidencias presentadas en este apartado muestran que Estefanía, Alexandra y Marcela establecieron conexiones entre las imágenes construidas en las dos situaciones. Así mismo, tal y como se mencionó en los apartados anteriores, aunque en la situación "rectángulo inscrito" las estudiantes reconocieron la noción de límite como una "tendencia", no consiguieron aceptar su existencia, en parte, por el valor de 0/0 que se presenta. En este aspecto, la simulación del movimiento de un vehículo, a través del software Modellus, fue un elemento fundamental para que ellas consiguieran aceptar la existencia de dicho límite.

Para iniciar el reconocimiento de la tasa variación instantánea, el investigador formuló la pregunta: ¿Cuál es la velocidad en dos? Las respuestas fueron diversas. Por ejemplo, Estefanía se mostró pensativa y respondió 16. Con ello mostró que estaba focalizada en la posición y no en la velocidad. Por su parte, Alexandra señaló que era necesario hacer los cálculos de nuevo con los triangulitos o sacar el límite; después de unos 
segundos, dijo con sorpresa, hey, ¿no sería también cuatro?; ante esto el investigador preguntó: ¿Por qué cuatro? Y de inmediato, Estefanía y Marcela argumentaron que porque era la misma ecuación y la misma "tendencia". Esto se convirtió en evidencia de un movimiento en las ideas construidas. Las estudiantes establecieron ciertas conclusiones como: En la situación № 1, cuando x (el segmento) se acercaba a dos, el cociente incremental del área con respecto al segmento se acercaba a cuatro. Es análogo a que, en la situación actual, cuando el tiempo se acerca a dos, la velocidad se acerca a cuatro.

En ese momento, el investigador le sugirió confrontar las experiencias desarrolladas en las tareas 1 y 2 . El investigador retomó la analogía planteada en el problema de movimiento del vehículo y generó el siguiente diálogo:

Investigador: Yo puedo preguntar, ¿cuál es la velocidad que lleva el carro en dos? Alexandra: Sí

Ante el silencio de tres compañeras, el investigador simula con su cuerpo el movimiento del vehículo, cuenta el tiempo y se detiene cuando dice "dos". Luego continúa:

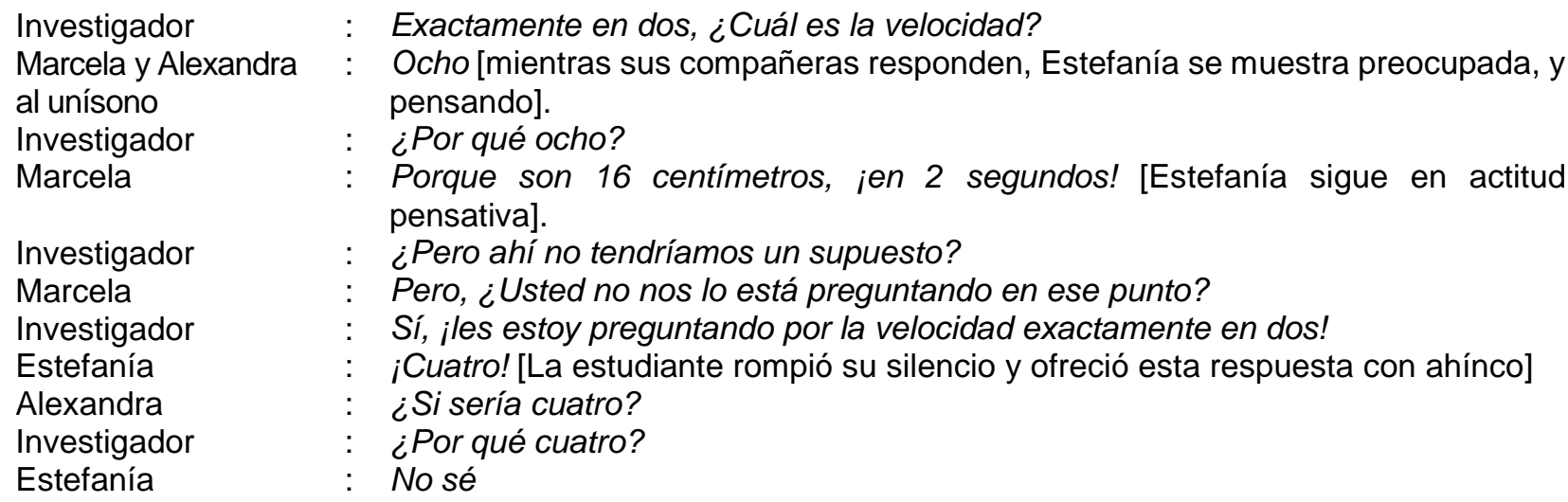

Se observa que tanto Marcela como Alexandra evocaron imágenes de la relación de proporcionalidad directa entre la posición y el tiempo, relación que revisaron una vez que el investigador afirmó que implicaba un supuesto. Unos segundos después del diálogo anterior, Alexandra tuvo ciertas imágenes mentales que fueron evidenciadas por el tono enérgico con el que verbalizó: Sí, es que es la tendencia. Y argumentó con el acercamiento por medio de intervalos. Sin embargo, Marcela no alcanzó a entender lo que Alexandra afirmó y replicó: ¿O sea que es cuatro? ¿Siempre va a ser cuatro? A lo que Alexandra le contestó: Cuando se acerca a dos, es cuatro; en otro punto sería otro valor.

Para apoyar las conclusiones de Alexandra, el investigador propuso analizar el cociente incremental a través del software y registrar su comportamiento en la tabla (Ver Fig. 3). Esta experiencia con el software fue determinante para que las estudiantes, en particular Marcela y Cristina, pudieran visualizar de nuevo la "tendencia" de la velocidad en el tiempo: 2 segundos. En el trabajo a seguir, se verificaron algunos valores cercanos a $\mathrm{t}=2$, y cómo la tasa de variación estuvo cada vez más cerca de cuatro; así confirmaron lo que se presenta en la tabla de valores. De nuevo, la imagen del límite apareció asociada a una "tendencia". Como evidencia de ello, las estudiantes verbalizaron mientras más me acerco a dos, la velocidad está más cerca de cuatro, pero en esta oportunidad, ante la pregunta por la velocidad en dos, las estudiantes ofrecieron la respuesta cuatro. La tasa de variación (velocidad) en $t=2$ era aceptada, a pesar de que no lo era en la tarea 1.

El siguiente diálogo se convierte en evidencia de este hecho:

Investigador

Estudiantes

Investigador

Estudiantes

Investigador

Estefanía y

Alexandra

Investigador

Estefanía

Alexandra

Estefanía

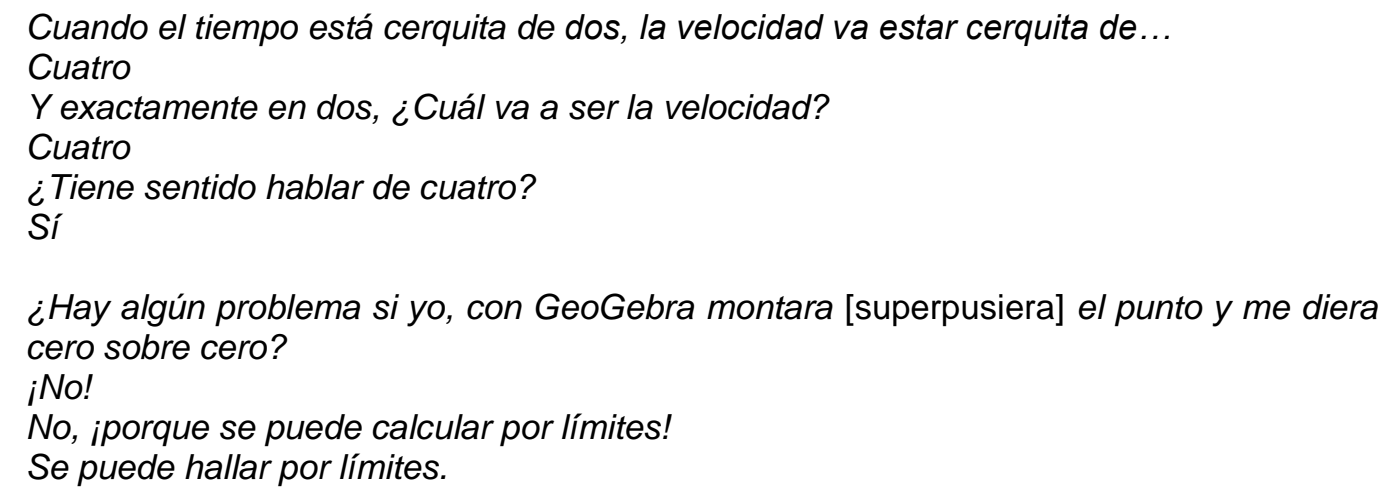




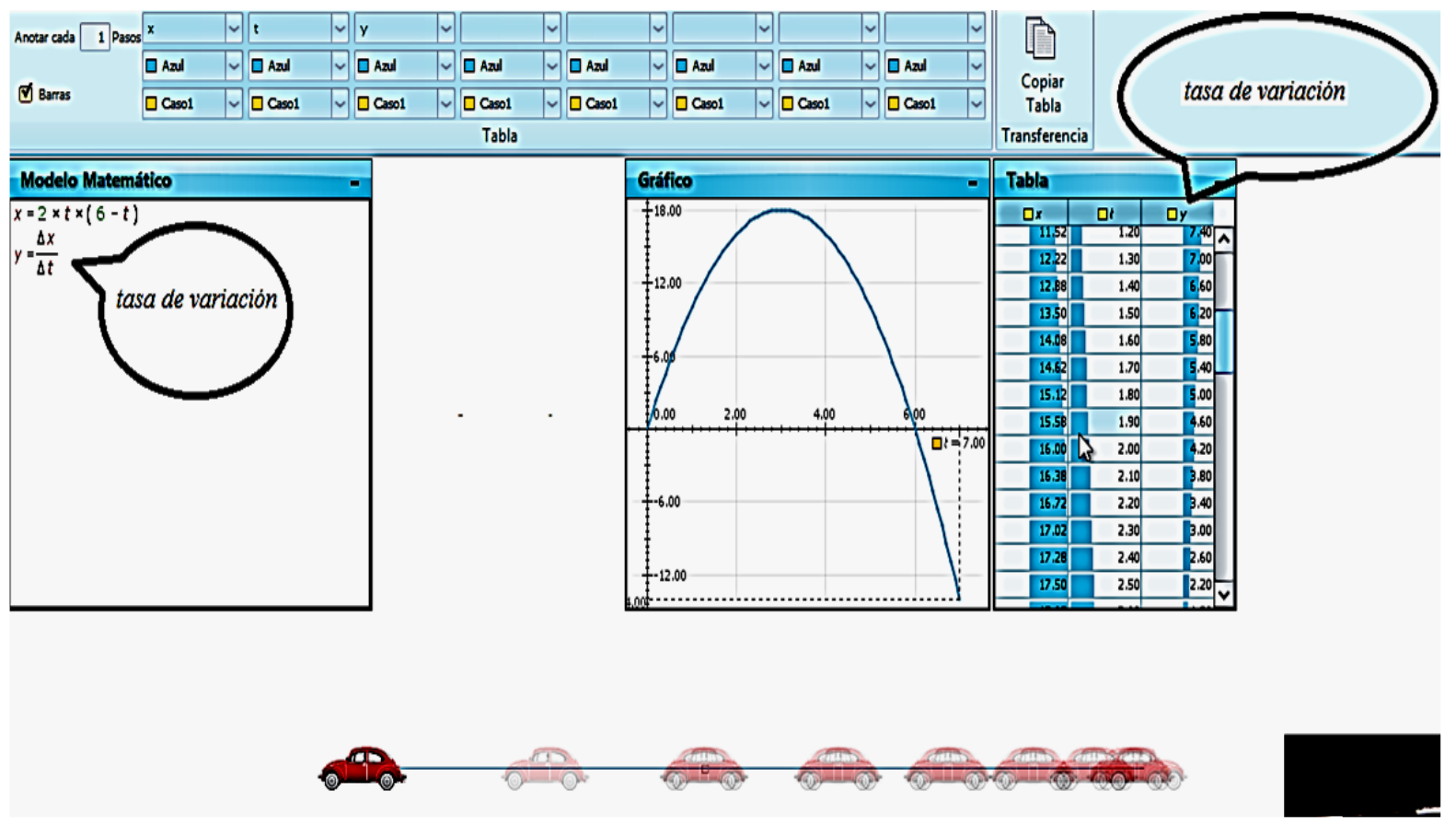

Fig. 3: Tasa de variación en el software Modellus

En este punto, el investigador hizo la reflexión con las estudiantes sobre "su resistencia a aceptar la existencia del límite, y que para ellas solo fuera una suposición”. Como respuesta, las estudiantes señalaron que sería como decir que en dos, el carro no tendría velocidad y es claro que sí la tiene. En el diálogo se evidencia además la diferencia que ellas pudieron establecer entre la manera de calcular la tasa de variación media y la necesidad de utilizar otros procedimientos (límites) para calcular la tasa de variación instantánea. Es estos episodios es evidente que las estudiantes consiguieron abstraer la existencia del límite, a partir de la abstracción de diferentes imágenes de la tasa de variación media. Este tipo de abstracciones les permitió reconocer la propiedad:

$$
\mathrm{Tv}_{\text {instantánea }}=\lim _{\Delta \mathrm{x} \rightarrow 0} \frac{\Delta \mathrm{y}}{\Delta \mathrm{x}}
$$

\section{DISCUSIÓN}

Un aspecto crítico en el estudio de la tasa de variación radica en los usos de los contextos y las representaciones (Herbert y Pierce, 2012; Vrancken y Engler, 2014). En el presente estudio se analizaron imágenes que las estudiantes fueron construyendo en dos contextos diferentes y el uso de representaciones kinestésicas, gráficas, algebraicas, numéricas y verbales. En las dos tareas, la modelación involucró el reconocimiento del fenómeno de variación, las cantidades (variables y constantes) que se involucran y las relaciones entre ellas para construir modelos que representan la covariación. A pesar de la similitud los procesos seguidos en ambas tareas, las imágenes que las estudiantes construyeron en el transcurso de cada proceso fueron diferentes.

El diseño de la primera tarea involucró el uso de 'texto dinámico' para disminuir la carga operatoria en el cálculo de la tasa de variación media. Al igual que los participantes del estudio de Ärlebäck et al. (2013), las estudiantes tuvieron una comprensión local y global de la tasa de variación media. La herramienta construida en el GeoGebra ofreció la posibilidad de calcular la tasa de variación media en un intervalo determinado (compresión local) pero también posibilitó el reconocimiento de una tendencia general a través del cálculo de la tasa de variación en tantos intervalos como las estudiantes quisieran (comprensión global). Esta tendencia contribuyó para que las estudiantes pudieran inferir el valor de la tasa de variación instantánea; sin embargo, también hizo que las estudiantes centraran más su atención en el cálculo aritmético y no tanto en la interpretación del resultado. Focalizarse en el cálculo aritmético pudo también deberse a la ausencia de un contexto que les posibilitara usar los significados construidos en sus experiencias cotidianas y extraescolares. Este hecho apoya la premisa de que la conexión entre conceptos va más allá del dominio matemático (Dolores y García-García, 2017), en particular, involucra conexiones de la estructura subyacente en las diferentes tareas, y sus conexiones con los fenómenos extramatemáticos.

La ausencia de un contexto 'realístico o auténtico' en la primera tarea también conllevó a que la interpretación de la tasa de variación (media e instantánea) no se asociara a una magnitud conocida; en esa tarea, la tasa 
de variación era una cantidad cuya medida se expresaba en " $\mathrm{cm}^{2} / \mathrm{cm}$ " y no una magnitud perceptible en la cotidianidad. Contrario a ello, en la segunda tarea, la tecnología además de recrear un fenómeno de movimiento también proporcionó significados de la tasa de variación como la velocidad de un móvil. Este significado fue fundamental para trascender las comprensiones de la tasa de variación instantánea. En cierta medida, los hallazgos de este estudio se relacionan con los de Herbert y Pierce (2011); sin embargo, el análisis de los episodios presentados muestra que los contextos usados para modelar, no permanecen neutros en la comprensión matemática. Este hecho ofrece evidencia adicional a los hallazgos de Villa-Ochoa y Berrío (2015) quienes rescatan los diferentes roles que tienen los contextos en la modelación matemática; asimismo, apoya la idea de incluir fenómenos de la experiencia de los estudiantes, en particular de la física, en el estudio de conceptos como el de derivada (Vrancken y Engler 2014).

Existen otros aspectos importantes que vale la pena resaltar. Un primer aspecto es que la tasa de variación media se interpretó en la primera tarea como la "comparación de dos estados" y se representó a partir de un triángulo rectánculo (cociente incremental). Esta representación fue dinámica en el sentido que podía dar cuenta de cualquier intervalo en cualquier punto; ello hizo que jugara un papel fundamental en la construcción de imágenes de la tasa de variación media, pues a través del ambiente con tecnología se ofrecieron insumos para coordinar la representación del fenómeno dinámico, la representacion geométrica, numérica y algebraica. Otro aspecto que se resalta tiene que ver con el diseño y el desarrollo de las tareas. A lo largo de la experiencia se propició el uso de descripciones cualitativas, la comparación aritmética de dos estados, la cantidad magnitud que describe el cambio promedio de una cantidad por unidad de cambio de la otra unidad. A pesar de ello, la experiencia proporcionada en la tarea 1 fue insuficiente para una comprensión de la tasa de variación instantánea y, como complemento, se requirió del estudio de un fenómeno que evocara los significados construidos en las experiencias extramatemáticas de las estudiantes. A partir de este estudio puede observarse que la comprensión de la tasa de variación instantánea demanda por parte del estudiante: (i) Superar la idea de límite como supuesto y (ii) Comparar y producir diferentes significados de los objetos matemáticos en diversos fenómenos y contextos. Estos resultados apoyan los resultados de Ärlebäck et al. (2013) y de Ärlebäck y Doerr (en prensa) quienes señalan que la comprensión matemática ocurre cuando los estudiantes desarrollan modelos matemáticos que pueden usarse y re-usarse en un rango de contextos estructuralmente similares.

Un aspecto adicional que se enfatiza es que las tecnologías utilizadas involucran la posibilidad de explorar y coordinar diferentes representaciones, visualizaciones y en el análisis de datos numéricos. Los resultados de este estudio muestran que todo ello se convirtió en insumo para la construcción de representaciones gráficas y algebraicas del modelo matemático. En los aspectos visuales, los hallazgos de este estudio ofrecen evidencia adicional a los elementos ya reportados por Rodríguez y Quiroz (2016), quienes con tecnologías digitales y fenómenos diferentes, también observaron que a través de la visualización se pueden establecer reflexiones y condiciones para construir modelos matemáticos a partir de los fenómenos recreados en cada tarea. Estos usos y aportes de las tecnologías apoyan los planteamientos de Borba y Villarreal (2005) quienes sugieren que la investigación cada vez demanda de usos más refinados de las tecnologías, que no conlleven a su "domesticación" para hacer con ellas lo que generalmente se haría con lápiz y papel.

Finamente, vale la pena resaltar que las tecnologías usadas en este estudio permitieron recrear fenómenos dinámicos y promover una comprensión de un objeto del cálculo acorde con la naturaleza dinámica de esta área del conocimiento (Tall, 2009; Parada et al., 2016). Así, el software Modellus también aportó, no sólo para recrear el ambiente de un movimiento uniforme y acelerado, sino también porque, mediante su manipulación, las estudiantes observaron relaciones entre cantidades como el recorrido y el tiempo; pero también aportó a que las estudiantes pudieran matematizar la velocidad como una tercera cantidad que, a su vez, ofreció significados del contexto para ampliar la comprensión de la tasa de variación instantánea. La coordinación de estas cantidades y el reconocimiento de la tasa de variación como una tercera cantidad son elementos claves dentro del razonamiento covariacional (Ärlebäck et al. 2013; Carlson et al., 2003)

\section{CONCLUSIONES}

La comprensión de la tasa de variación media e instantánea es fundamental en el razonamiento covariacional y la comprensión de la derivada (Ärlebäck y Doerr, en prensa; Vrancken y Engler, 2014). En su marco conceptual Carlson et al. (2003) señalan que la comprensión de la tasa de variación instantánea "incluye una consciencia de que la razón de cambio instantánea resulta de refinamientos más y más pequeños en la razón de cambio promedio" (p. 129); sin embargo, evidencias empíricas muestran que estas acciones son insuficientes para lograr una compresión de ella (Villa-Ochoa, 2012).

Los hallazgos presentados en este artículo muestran que existen estudiantes para quienes la secuencia de refinamientos más pequeños en la tasa de variación media no es suficiente para comprender la tasa de variación instantánea. En ese sentido, este artículo aporta evidencia adicional frente a otros aspectos que 
intervienen en la transición entre los niveles de razonamiento asociados a estas dos tasas de variación. En coherencia con ello, este estudio pone a consideración aspectos a través de los cuales los marcos que se encargan de estudiar esta transición podrían extenderse.

Como una manera de atender al propósito declarado en este artículo, se destaca que las tecnologías digitales, los contextos y los fenómenos que se modelaron no fueron neutros en la transición hacia la tasa de variación instantánea. A través del software GeoGebra se implementó una tarea que recreó un fenómeno dinámico y el estudio aritmético de la tasa de variación media en intervalos cada vez más pequeños. Si bien es cierto que la tarea fue fundamental en el proceso de estudio y modelación de la variación y en la coordinación de representaciones; también es cierto que al centrar la atención en la secuencia aritmética las estudiantes pudieron conjeturar un valor para la tasa de variación instantánea, pero no aceptaron su existencia.

Para poder aceptar la existencia de la tasa de variación instantánea fue fundamental la conjunción de un contexto propio de la experiencia de las estudiantes y la posibilidad de experimentar, representar y confrontar contextos a través de la tecnología. En la aceptación de la existencia de ese valor aportaron tanto los significados que la velocidad en un tiempo determinado tenía en la experiencia de las estudiantes como la confrontación de las estructuras subyacentes en las dos tareas de modelación. En la conjunción entre modelación y tecnologías se constituyó un sistema de experiencias, significados y representaciones a través de los cuales la tasa de variación instantánea cobró sentido para las estudiantes.

\section{REFERENCIAS}

Arcavi, A., Modelling with graphical representations, http://flm-journal.org/, ISSN: 0228-0671, For the learning Mathematics, 28(2), 2-10 (2008)

Ärlebäck, J., Doerr, H. y O'Neil, A., A Modeling Perspective on Interpreting Rates of Change in Context, doi: 10.1080/10986065.2013.834405, Mathematical Thinking and Learning, 15(4) 314-336 (2013)

Ärlebäck, J. B., y Doerr, H. M., Students' interpretations and reasoning about phenomena with negative rates of change throughout a model development sequence, doi 10.1007/s11858-017-0881-5, ZDM - Mathematics Education, First Online, 19 August (2017)

Arrieta Vera, J., y Díaz Moreno, L., Una perspectiva de la modelación desde la Socio epistemología, doi: 10.12802/relime.13.1811, Revista Latinoamericana de Investigación en Matemática Educativa, 18(1), 19-48 (2015)

Báez, A., Martínez-López, Y., Pérez, O. y Pérez, R., Propuesta de tareas para el Desarrollo del Pensamiento Variacional en Estudiantes de Ingeniería, doi: 10.4067/S0718-50062017000300010, Formación Universitaria, 10(3), 93-106 (2017)

Blum, W., Can Modelling Be Taught and Learnt? Some Answers from Empirical Research, doi: 10.1007/978-94-007-09102_3, In G. Kaiser, W. Blum, R. Borromeo F., y G. Stillman (Eds.), Trends in Teaching and Learning of Mathematical Modelling, 15-30, Dordrecht: Springer (2011)

Borba, M. y Villarreal, M., Humans-with-Media and the reorganization of mathematical thinking, doi: 10.1007/b105001, New York: Springer (2005)

Bosch, M., García, F., Gascón, J. e Higueras, L., La modelización matemática y el problema de la articulación de la matemática escolar, http://www.revista-educacion-matematica.org.mx/revista/, ISSN: 1665-5826, Educación Matemática, 18(2), 37-74 (2006)

Cabezas, C. y Mendoza, M., Manifestaciones Emergentes del Pensamiento Variacional en Estudiantes de Cálculo Inicial, doi: 10.4067/S0718-50062016000600003, Formación Universitaria, 9(6), 13-26 (2016)

Carlson, M., Jacobs, S., Coe, E., Larsen, S. y Hsu, E., Razonamiento covariacional aplicado a la modelación de eventos dinámicos: un marco conceptual y un estudio, http://funes.uniandes.edu.co/1520/, ISSN: 0122-5057, Revista EMA, 8(2), 121-156 (2003)

Daher, W. y Shahbari, J., Pre-service teachers' modelling processes through engagement with model eliciting activities with a technological tool, doi: 10.1007/s10763-013-9464-2, International Journal of Science and Mathematics Education, 13(1), 25-46 (2015)

Dolores Flores, C. y García-García, J., Conexiones Intramatemáticas y Extramatemáticas que se producen al Resolver Problemas de Cálculo en Contexto: un Estudio de Casos en el Nivel Superior, doi 10.1590/1980-4415v31n57a08, Bolema: Boletim de Educação Matemática, 31(57), 158-180 (2017)

Gordillo, W., y Pino-fan, L.R., Una Propuesta de Reconstrucción del Significado Holístico de la Antiderivada, doi: 00.1590/1980-4415v30n55a12, Bolema: Boletim de Educação Matemática, 30(55), 535-558 (2016)

Herbert, S. y Pierce, R., What is rate? Does context or representation matter? doi: 10.1007/s13394-011-0026-z, Math. Ed. Res. J., 23(4), 455-477 (2011)

Herbert, S. y Pierce, R., Revealing educationally critical aspects of rate, doi: 10.1007/s10649-011-9368-4 Educ. Stud. Math., 81(1), 85-101 (2012) 
Kaiser, G. y Sriraman, B., A global survey of international perspectives on modelling in mathematics education, doi: 10.1007/BF02652813, ZDM-Mathematics Education, 38(3), 302-310 (2006)

Parada, S., Conde, L. y Fiallo, J., Mediación Digital e Interdisciplinariedad: una Aproximación al Estudio de la Variación, doi: 10.1590/1980-4415v30n56a10, Bolema: Boletim de Educação Matemática, 30(56), 1031-1051 (2016)

Rodríguez, R. y Quiroz, S., El papel de la tecnología en el proceso de modelación matemática para la enseñanza de las ecuaciones diferenciales, doi: 10.12802/relime.13.1914, Rev. Latinoamericana de Investigación en Matemática Educativa, 19(1), 99-124 (2016)

Soares, D. y Borba, M., The role of software Modellus in a teaching approach based on model analysis, doi: 10.1007/s11858-013-0568-5, ZDM - Mathematics Education, 46(4), 575-587 (2014)

Suarez, L., Modelación-Graficación para la matemática escolar, ISBN: 978-84-9969-614-0, México D.F.: Díaz de Santos (2014)

Tall, D., Dynamic mathematics and the blending of knowledge structures in the calculus, doi: 10.1007/s11858-009-01926, ZDM - Mathematics Education, 41(4), 481-492 (2009)

Villa-Ochoa, J. A. y Berrío, M., Mathematical Modelling and Culture: An Empirical Study, In G. A. Stillman, W. Blum, y M. Biembengut (Eds.), doi: 10.1007/978-3-319-18272-8, Mathematical Modelling in Education Research and Practice 241250. Switzerland: Springer International Publishing (2015)

Villa-Ochoa, J. A., Razonamiento covariacional en el estudio de funciones cuadráticas, https://goo.gl/CeLj3n, ISSN: 0121 3814, Tecné, Episteme y Didaxis: TED (31), 9-25 (2012)

Villa-Ochoa, J. A., Modelación matemática a partir de problemas de enunciados verbales: un estudio de caso con profesores de matemáticas, doi: 10.11144/Javeriana.m8-16.mmpe, Magis, Rev. Internacional de Investigación en Educación, 8(16), 133-148 (2015)

Vrancken, S., y Engler, A., Una introducción a la derivada desde la variación y el cambio: resultados de una investigación con estudiantes de primer año de la universidad, doi 10.1590/1980-4415v28n48a22, Bolema: Boletim de Educação Matemática, 28(48), 449-468 (2014)

Yin, R., Case study research, Design and methods (4th Ed.), ISBN: 9781412960991, Thounsand Oaks, CA: Sage Publications (2009) 